THP-33(T5)

\title{
Optical biopsy of lung adenocarcinoma using multiphton fluorescence and harmonic generation microscopy
}

\author{
Chun-Chin Wang , Feng-Chieh Li , Wen Lo, Chen-Yuan Dong* \\ Microscopic Biophysics Laboratory, Department of Physics, National Taiwan University, \\ Taipei, Taiwan 106, ROC
}

*To whom correspondence should be addressed : cydong@phys.ntu.edu.tw

Tel.: 886-2-3366-5155 ; Fax : 886-2-3366-5244

\begin{abstract}
Lung tumor is a major disease in Taiwan and the development of minimally invasive imaging to detect and diagnose lung tumor in vivo is of biomedical value. ${ }^{[1][2]}$ However, prior to in vivo investigation, it is important to obtain the multiphoton signatures associated with lung tumor ex vivo. In this investigation, we used multiphoton microscopy to image normal and cancerous lung tissues (adenocarcinoma). Our results show that a combination of harmonic generation and multiphoton autofluorescence imaging may be used to acquire structural information in discerning lung carcinoma from normal lung tissues.
\end{abstract}

\section{INTRODUCTIONS}

Since the development of multiphoton microscopy in the 1990s, a number of important fields of biomedical research has benefited from this imaging modality. However, disease diagnosis using non-linear microscopy has been limited due to the limitation in imaging depths of optical microscopy. Since lung tumor is a major disease, we are pursuing the possibilities of using multiphoton microscopy to diagnose lung tumors in vivo. To prepare for such investigations, we need to differentiate lung tumor from normal tissues ex vivo. In this investigation, we imaged human lung carcinoma and compared the results to images of normal tissues.

\section{METHODS AND MATERIALS}

The multiphoton microscope used in this study is based on an upright microscope (E800, Nikon, Japan). A diode-pumped (Millennia X, Spectra Physics, Mountain View, CA), titanium-sapphire laser (Tsunami, Spectra Physics) was used as the excitation source. The $780 \mathrm{~nm}$ output of the laser system was used to induce second harmonic generation (SHG) and autofluorescence signals from the lung tissue specimens. A dual channel detection mode was used for simultaneous image acquisition.

\section{RESULTS}

The multiphoton images of normal and adenocarcinoma lung tissues are shown in Fig.2 and Fig.3, respectively.

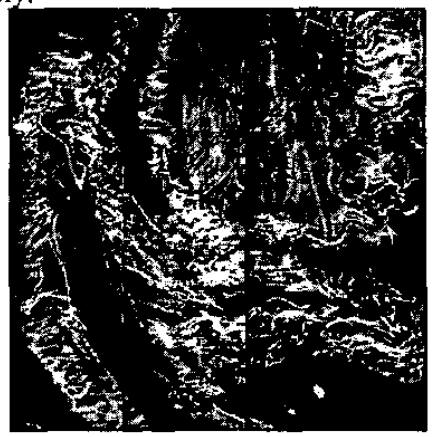

Fig. 2 Multiphoton image of normal human lung tissue (blue: SHG, green: autofluorescence).

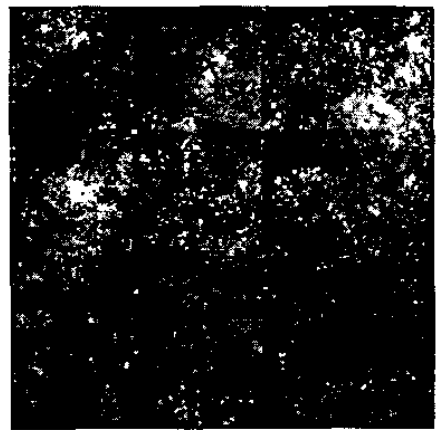

Fig. 3 Multiphoton image of human lung adenocarcinoma (blue: SHG, green: autofluorescence). 


\section{THP-33(T5)}

From the images, it is evident that the SHG and autofluroescence images of normal tissue show well structures fiber structures. On the other hand, lung adecinorma tissue shows a much less organized SHG fiber pattern with masses of autofluorescent cells.

\section{CONCLUSIONS}

The multiphoton images we have acquired show that normal and lung adenocarcinoma tissue may be discerned by a combination of second harmonic and autofluorescence imaging. This study demonstrates that with the appropriate development of in vivo imaging technologies, multiphoton microscopy may be used to diagnose and investigate lung tumors in vivo.

\section{REFERENCES}

[1] B. Kulapaditharom, V. Boonkitticharoen, "Performance characteristics of fluorescence endoscope in detection of head and neck cancers." Annals of otology rhinology and laryngology, 110(1), pp. 45-52(2001).

[2] J.D. Pitts, R.D. Sloboda, K.H. Dragnev, et al, "Autofluorescence characteristics of immortalized and carcinogen-transformed human bronchial epithelial cells." Journal of biomedical optics, 6(1), pp. $31-40(2001)$.

[3] P.So, C.Y. Dong, B.R. Masters, et al. "Two-photon excitation fluorescence microscopy. " Annual Review of BiomedicalEengineering, 2,pp. 399-429(2000). 